\title{
Performance Evaluation of a Morphing Trailing Edge Using Multipoint Aerostructural Design Optimization
}

\author{
David A. Burdette * \\ Gaetan K.W. Kenway ${ }^{\dagger}$ \\ Joaquim R. R. A. Martins ${ }^{\ddagger}$ \\ University of Michigan, Ann Arbor, Michigan, 48109, United States
}

\begin{abstract}
New configurations and technologies like adaptive morphing trailing edges offer the potential to improve the fuel efficiency of commercial transport aircraft. Using modern computational tools, it has become possible to effectively analyze the extent to which this technology improves aircraft performance. Parametrizing high fidelity structural models coupled to RANS-based aerodynamics with hundreds of variables provides the accuracy necessary to complete a meaningful comparison of aircraft with an without morphing wing technology. Using this computational approach, we perform multipoint aerostructural optimization, to provide an objective function which can leverage the adaptability provided by wing morphing. We show that for a seven point mulitpoint, the addition of a morphing trailing edge device along the aft $40 \%$ of the wing can reduce cruise fuel burn by more than $5 \%$. A large portion of the savings produced by morphing trailing edges result from a significant reduction in structural weight, enabled by adaptive maneuver load alleviation. We also show that a smaller morphing device along the aft $30 \%$ of the wing produces nearly as much fuel burn reduction as the larger morphing device, and that morphing technology is particularly effective for high aspect ratio wings.
\end{abstract}

\section{Introduction}

Increased awareness of environmental concerns and fluctuations in fuel prices in recent years have led the aircraft manufacturing industry to push for improved aircraft fuel efficiency. Increases in fuel efficiency have been moderate in recent decades as compared to the increases seen between 1960 and 1990, as decades of experience and optimization have left only relatively small improvements to be made on conventional wing and tube configurations. This combination of increased interest in reducing fuel burn and the recent plateau of fuel burn improvements with a conventional configuration has pushed aircraft manufacturers to consider new technologies and configurations that offer the potential for further improvements in fuel efficiency. In the long term, new configurations such as the blended wing body (BWB) or truss braced wing (TBW) offer promising potential; however, they are likely a few decades from commercial availability. There are also a number of technologies which are closer to entering the market that offer efficiency improvements on conventional configurations, like tow steered composites and functionally graded materials [1]. Another such technology is adaptable morphing trailing edges. Companies such as FlexSys have already developed such devices [2,3] and have begun flight tests in collaboration with NASA and the U.S. Air Force Research Lab [4]. This technology offers the potential to create wings that can adapt to flight conditions, enabling engineers to design the wing shape and sizing not as a compromise between good performance at a series of flight conditions, but rather for near-optimal performance at a wide variety of conditions. In order to determine the value of such a device, the benefit of that freedom to design for a variety of flight conditions must be quantified.

Various studies have explored the benefits of morphing trailing edges in the past. In the late nineties, Hanselka [5] and Monner et al. [6] outlined the aerodynamic benefits associated with morphing trailing edge devices, and offered designs for morphing mechanisms. More recently, Molinari et al. [7] explored the potential of the technology using a multidisciplinary optimization approach considering mission, aerodynamic, materials, and structural disciplines. That work used low fidelity models and therefore was unable to capture the effects of small shape changes, which have been shown to be crucial in transonic aerodynamic performance [8]. Reynolds averaged Navier-Stokes based simulations more effectively capture the intricate flow phenomena that have a substantial impact on the performance of a wing and have been used for analysis of an adaptive trailing edge [9]. In that work, Lyu and Martins found that a morphing trailing edge can provide drag reductions of between one and five percent, depending on the flight

\footnotetext{
*Ph.D. Candidate, Department of Aerospace Engineering, AIAA Student Member

${ }^{\dagger}$ Research Investigator, Department of Aerospace Engineering

${ }^{\ddagger}$ Professor, Department of Aerospace Engineering, AIAA Associate Fellow
} 
condition. Wakayama et al. [10] found similar results in theri work considering morphing devices on three different aircraft configurations. The authors previously used high fidelity coupled aerostructural optimization to evaluate a wing with a morphing trailing edge at a single cruise point [11]. That work showed the ability of the morphing trailing edge to drastically effect the wing's lift distribution, but in this work we seek to build off of these previous results by expanding the number of flight conditions where the performance of the wing is considered. The main advantage of morphing trailing edge technology is its ability to adapt a wing to changing flight conditions, so we expect multipoint analysis to provide better opportunities for the morphing trailing edge to improve performance. The inclusion of structural analysis is also important, as structural deflections will vary at different flight conditions, providing further opportunity for the morphing trailing edge to improve performance.

The paper is organized as follows: Section II describes the computational tools used in this work. Section III describes multipoint aerostructural optimizations of the uCRM configuration with a morphing trailing edge. Three point and seven point stencils are considered. reducing fuel burn. Finally, Section IV outlines the significance of this work and details the impact this technology can have on the airline industry.

\section{Overview of numerical methods}

In this section, we discuss the numerical analysis and design optimization algorithms used in this work. The numerical algorithms are implemented in components of the MACH (MDO for Aircraft Configurations with High fidelity) framework [12].

\section{A. Geometric parametrization}

Geometric shape changes are parametrized using a Free Form Deformation (FFD) approach [13], a technique that is also used frequently in computer graphics to generate deformations of solid geometries [14]. The approach implants the solid geometry within an outer hull, which is parametrized with a series of control points. The control points generate deformations of the encompassing volume, which are interpolated onto the geometry. The interpolation generates a region of influence spanning two control points in each (i, j, and $\mathrm{k}$ ) parametric direction, and provides smooth deformations that are defined with a relatively small number of design variables. Aggregating control points also allows for the creation of larger-scale global design variables such as chord, span, and twist. An example of an FFD used for a morphing trailing edge optimization is shown in Figure 1.

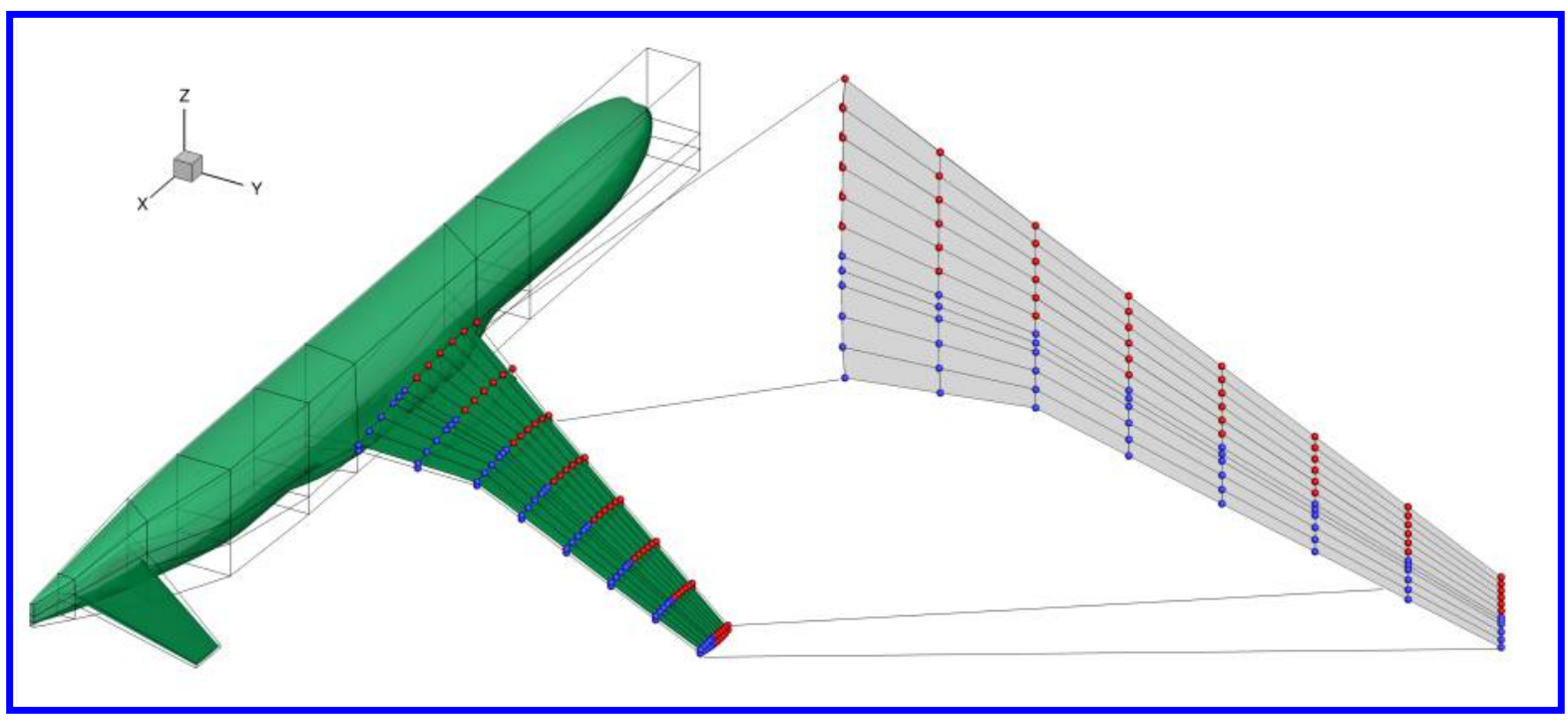

Figure 1: This FFD is designed such that control points are aligned with 30,35 , and $40 \%$ of the chord. Providing freedom to the 4 aft-most rows of design variables in this case would result in geometric deformations-and thus a morphing device-spanning the area enclosed by the blue control points.

Note that the FFD does not have a uniform distribution of control points along the (chord-wise) $x$-direction. Instead, there is a grouping of control points near the leading edge of the morphing device. This control point distribution 
allows for simple implementation of the morphing trailing edge. The subset of the FFD control points on the aft region of the wing is given additional freedom at each flight condition, allowing the wing to assume different shapes at different flight conditions. Since we know that the geometric parametrization restricts deformations to a region exactly spanning 2 control points in each direction, we can define a morphing boundary simply through control point alignment. The additional rows of control points near that boundary provide a means to generate smooth and rapid transitions between the morphing and non-morphing regions.

The free form deformation implementation used in this work also supports the creation and usage of sub-FFDs, such as that used for the horizontal tail. A sub-FFD around the tail allows the optimizer to generate solid body rotation of the horizontal stabilizer, which is important for trimming the aircraft. A sub-FFD could have also been used to parametrize the morphing device, but the use of configuration-specific design variables supported in this FFD implementation is more straightforward. This feature generates a fully populated set of design variables visible to all configurations. However, a subset of the design variables can be associated with specific flight conditions. If the FFD is generating deformations for a flight condition other than that associated with the set of design variables, then those displacements are not applied to the FFD.

\section{B. Mesh deformation}

The nature of morphing trailing edge deformations makes mesh deformation a challenge, particularly during maneuver conditions where the mesh needs to deform according to both low frequency structural deformations and high frequency deformations from the morphing trailing edge. As such, an inverse-distance-weighting warping algorithm similar to that suggested by Luke et al. [15] is used in this work. The warping scheme interpolates both displacements and rotations of the surface into the volume mesh. One of the principal strengths of this approach is its ability to preserve mesh perpendicularity near surfaces. This feature is particularly valuable in the context of morphing trailing edge optimizations, for which past experience has shown that other mesh warping schemes often generate negative cell volumes and mesh crossover near the deformed trailing edge. Computational efficiency is achieved through the use of a tree-like data structure and an efficient spatial search algorithm.

\section{CFD solver}

The aerodynamic solver within the MACH framework is SUMad (formerly SUmb) [16], a finite volume CFD solver for structured multiblock meshes. In order to provide sufficient fidelity for shape optimization of a transonic wing, SUMad solves the Reynolds averaged Navier-Stokes (RANS) equations, with a one equation Spalart-Allmaras (SA) turbulence model. The flow is solved with a combination of Runge-Kutta (RK) and Newton-Krylov (NK) schemes. SUMad also computes gradients of the functions and constraints, using a discrete adjoint approach, with partial derivatives computed with a combination of analytic and reverse mode automatic differentiation (AD) techniques [17]. With this implementation, the cost of computing a gradient is nearly independent of the number of design variables. A matrix-free method for the adjoint computation has been implemented, which reduces the memory requirements.

\section{Structural solver}

The structural solver within the MACH framework is the Toolkit for the Analysis of Composite Structures (TACS) [18]. TACS computes displacements, stresses, and their derivatives. The challenge for transport aircraft structural problems involving shell elements is the poor matrix conditioning. Matrix condition numbers exceeding $\mathcal{O}\left(10^{9}\right)$ are not uncommon within this context. A Schur complement method is used in the structural solution, and again the adjoint method is used in the gradient computations.

\section{E. Coupled aerostructural solver}

The main role of the aerostructural solver is to couple the aerodynamic and structural solvers, SUMad and TACS. Structural deformations are transferred to the aerodynamic mesh using a rigid link method similar to that proposed by Brown [19], in which the nodes of the aerodynamic mesh are associated with the nearest point of the structural model. The deformation of those points in the structure are then applied to their associated points in the aerodynamic mesh. A consistent force vector is constructed from the integrated aerodynamic forces, and applied to the structure. The aerostructural solver then solves the coupled nonlinear system of equations. As is the case with both of its components, the aerostructural solver also computes derivatives using the adjoint method [12]. 


\section{F. Optimization method}

The optimizations in this work are performed using SNOPT (Sparse Nonlinear OPTimizer) [20], an optimization algorithm that uses a sequential quadratic programming (SQP) approach. This optimization method is desirable due to its relatively small required number of function and gradient calculations, which is particularly important for aerostructural optimization where both function analyses and gradient computations are expensive. Function and gradient values are provided to SNOPT and the Hessian of the Lagrangian is generated using a quasi-Newton approximation. SNOPT was interfaced using a sparse implementation of pyOPT [21], a Python package that allows for rapid formulation of nonlinear optimization problems.

\section{Multipoint aerostructural optimization}

To investigate the effectiveness of a morphing trailing edge device in reducing the fuel burn of a commercial transport aircraft, we performed a number of multipoint aerostructural optimizations using the tools outlined in Section II. We previously performed single point aerostructural optimizations with and without a morphing trailing edge [11]. That work showed limited improvements with the addition of a morphing trailing edge, as the non-morphing wing had nearly optimal for that single flight condition. This was because the only compromises the conventionally designed wing had to make to its performance were to prevent structural failure at $2.5 \mathrm{~g}$. In reality, wings need to be designed to perform well at a variety of flight conditions, not only at a single cruise condition. Optimizing a wing for performance at multiple flight conditions addresses this issue. We start by discussing two baseline optimizations in Section III.A. In the following two subsections, we consider two variations on those baseline optimizations. We explore the effects of reducing the size of the morphing device, and then examine whether morphing trailing edge devices are more effective for higher-aspect ratio wings.

\section{A. Baseline uCRM optimizations}

The initial configuration for the baseline optimizations is the undeformed Common Research Model (uCRM) [22]. This configuration provides a jig shape and structure that deforms to the the shape of the original CRM [23] at $1 \mathrm{~g}$. The initial wing is first optimized without any morphing capabilities, to provide a fair reference from which to measure the improvements provided in subsequent optimizations. Those subsequent optimizations include morphing variables at all of the non-nominal flight conditions. Comparing the fuel burn of the optimized aircraft with and without the morphing design variables, we isolate and quantify the effects of the morphing trailing edge.

We consider two multipoint stencils: a three-point stencil with varying lift coefficient and a seven point stencil with varying lift coefficient, Mach number, and altitude. The nominal flight condition for the uCRM is at a Mach number of 0.85 and a lift coefficient $\left(C_{L}\right)$ of 0.5 . The multipoint stencils are centered around this nominal flight condition. The Bregeut range equation is used to approximate the fuel burn of each configuration in the multipoint stencil. The average of the fuel burn at each of the conditions is the objective function of the optimization. Maneuver conditions at 2.5 and $-1.0 \mathrm{~g}$ are considered to appropriately size the members of the wingbox. Stress and buckling constraints are added for both maneuver conditions [24]. The multipoint stencils are described in more detail in Tables 1 and 2 below. The altitude variations in the 7-point stencil were selected to correspond to full and empty aircraft weights.

\begin{tabular}{llll}
\hline Flight condition & Mach & $C_{L}$ & Altitude (ft) \\
\hline Nominal Cruise & 0.85 & 0.50 & 34,000 \\
Low $C_{L}$ Cruise & 0.85 & 0.45 & 34,000 \\
High $C_{L}$ Cruise & 0.85 & 0.55 & 34,000 \\
\hline
\end{tabular}

Table 1: Overview of the 3 point stencil.

The objective of each of the optimizations is to minimize the average fuel burn over each of the flight conditions in the multipoint stencil. To do this, the optimizer adjusts a wide variety of design variables. The angle of attack can change at each flight condition (cruise and maneuver), so that each lift constraint is satisfied. The tail rotation angle is adjusted to trim the aircraft. The shape of the wing is controlled through adjustments of the FFD, which are achieved in a variety of ways. There are 192 shape design variables, which define the non-morphed, nominal optimized wing shape. These variables are available in each of the four optimizations. They adjust the $z$-location of control points only, preserving the planform of the aircraft. A subset of 64 shape variables defines the morphing device. As such, 


\begin{tabular}{llll}
\hline Flight condition & Mach & $C_{L}$ & Altitude (ft) \\
\hline Nominal Cruise & 0.85 & 0.50 & 34,000 \\
Low $C_{L}$ Cruise & 0.85 & 0.45 & 34,000 \\
High $C_{L}$ Cruise & 0.85 & 0.55 & 34,000 \\
Low $M$ Cruise & 0.82 & 0.50 & 34,000 \\
High $M$ Cruise & 0.88 & 0.50 & 34,000 \\
Low alt. Cruise & 0.85 & 0.50 & 30,000 \\
High alt. Cruise & 0.85 & 0.50 & 40,000 \\
\hline
\end{tabular}

Table 2: Overview of the 7-point stencil.

64 variables are added for each non-nominal flight condition. For the 3-point stencil, this results in 256 additional shape variables: 64 for each of the two additional cruise conditions and the two maneuver conditions. Wing twist variables are also defined (as aggregate movements of control points) at eight spanwise locations, to give the optimizer more direct control of the twist distribution. Shape changes are limited by a variety of constraints. The volume of the wing is constrained not to decrease, as to ensure sufficient space for fuel. At 20 spanwise locations, the leading edge and trailing edge thickness is constrained not to decrease, to provide low speed performance and manufacturability, respectively. Additional thickness constraints provide room for mounting actuation mechanisms to the aft spar, but limit shape changes in the morphing region. Shear twist is avoided by constraining the movements of the leading and trailing edge control points. Finally, 854 structural variables allow the optimizer to adjust thicknesses of spars, skins, ribs, and stiffeners. Length variables are also provided to the structural model, but they are constrained to be consistent with the geometric lengths through a series of nonlinear consistency constraints. The structure is constrained not to buckle at either maneuver condition, and is constrained not to fail at the $2.5 \mathrm{~g}$ pull up condition. These constraints are aggregated using KS functions, to limit the number of required adjoint solutions. Finally, 696 linear adjacency constraints ensure that thicknesses do not change by more than $5 \mathrm{~mm}$ between adjacent components of the structure. A summary of the four optimization problems is shown in Table 3. 


\begin{tabular}{|c|c|c|c|c|c|c|}
\hline & Function/variable & Description & 3C & 3M & 7C & 7M \\
\hline minimize & Fuel burn & & & & & \\
\hline \multirow[t]{8}{*}{ w.r.t. } & $x_{\alpha_{\mathrm{c}}}$ & Angle of attack at cruise & 3 & 3 & 7 & 7 \\
\hline & $x_{\alpha_{\mathrm{m}}}$ & Angle of attack at maneuver & 2 & 2 & 2 & 2 \\
\hline & $x_{\text {shape }}$ & Full wing FFD control pts & 192 & 192 & 192 & 192 \\
\hline & $x_{\text {morph }}$ & Morphing TE FFD control pts & 0 & 256 & 0 & 512 \\
\hline & $x_{\text {twist }}$ & Wing twist & 8 & 8 & 8 & 8 \\
\hline & $x_{\text {tail }}$ & Tail rotation angle & 5 & 5 & 9 & 9 \\
\hline & $x_{\text {struct }}$ & Structural sizing variables & 854 & 854 & 854 & 854 \\
\hline & & Total DVs & 1064 & 1320 & 1072 & 1584 \\
\hline \multirow[t]{13}{*}{ subject to } & $L=n_{i} W$ & Lift & 5 & 5 & 9 & 9 \\
\hline & $M=0$ & Pitching moment & 5 & 5 & 9 & 9 \\
\hline & $V / V_{\text {init }} \geq 1$ & Fuel volume & 1 & 1 & 1 & 1 \\
\hline & $t /\left.t_{\text {init }}\right|_{\text {LE }} \geq 1$ & Leading edge thickness & 20 & 20 & 20 & 20 \\
\hline & $t /\left.t_{\text {init }}\right|_{\mathrm{TE}} \geq 1$ & Trailing edge thickness & 20 & 20 & 20 & 20 \\
\hline & $t /\left.t_{\text {init }}\right|_{\text {spar }} \geq 1$ & Morphing region thickness & 20 & 220 & 20 & 220 \\
\hline & $\Delta z_{\mathrm{LE}_{\text {upper }}}=-\Delta z_{\mathrm{LE}_{\text {lower }}}$ & Fixed leading edge & 8 & 8 & 8 & 8 \\
\hline & $\Delta z_{\mathrm{TE}_{\text {upper }}}=-\Delta z_{\mathrm{TE}_{\text {lower }}}$ & Fixed trailing edge & 8 & 0 & 8 & 0 \\
\hline & $L_{\text {panel }}-x_{\text {panel }}=0$ & Consistency constraints (NL) & 272 & 272 & 272 & 272 \\
\hline & $\mathrm{KS}_{\text {stress }} \leq 1$ & Maneuver stress & 3 & 3 & 3 & 3 \\
\hline & $\mathrm{KS}_{\text {buckling }} \leq 1$ & Maneuver buckling & 6 & 6 & 6 & 6 \\
\hline & $\left|x_{\text {struct }_{i}}-x_{\text {struct }_{i+1}}\right| \leq 5 \mathrm{~mm}$ & Linear adjacency constraints & 696 & 696 & 696 & 696 \\
\hline & & Total constraints & 1064 & 1256 & 1072 & 1264 \\
\hline
\end{tabular}

Table 3: Overview of the 3 point conventional (3C), 3 point morphing (3M), 7 point conventional (7C), and 7 point morphing (7M) optimization problems.

Having outlined the setup of the optimization problems, we now describe the results of the optimizations. We start by considering the results for the 3-point optimization. Figure 2 shows a comparison of the two optimizations (conventional and with morphing). The addition of morphing had a clear positive effect on the performance of the wing, as the average fuel burn was reduced by $2.53 \%$. This reduction seems to be largely due to a substantial $22.4 \%$ reduction in structural weight. Looking at the pressure contours on the top of the wing for each of the cruise flight conditions, we see that in both optimizations there are few shocks and the pressure distribution is consistent with optimal transonic results with a smooth pressure recovery [25]. On the front view of the aircraft, we see displaced wing shapes at the nominal cruise case as well as both maneuver conditions. We see that the addition of morphing at the maneuver conditions reduced the wing displacements at maneuver, which is consistent with the structural weight reduction we mentioned before. To see how this is achieved, we refer to the lift distribution below the front view of the aircraft. The distributions at the nominal cruise case and the $2.5 \mathrm{~g}$ maneuver overlay an elliptical lift distribution (in gray). The wing with morphing is able to shift more of the maneuver load inboard, reducing the root bending moment on the wing, which results in a much lighter structure. Below that we see twist distributions, which show that the conventional wing washes out the tip using aeroelastic coupling at maneuver, while the wing with morphing produces a twist distribution more closely matching that at cruise. This is because adjustable camber handles the inboard shift of the load for the wing with morphing. The thickness distribution of the structural members shows that the structure is thinner almost everywhere (where it is not limited by minimum gauge thickness) with the addition of morphing. The structural failure contours show that adding morphing allows the optimizer to push more structural members closer to their failure point, spreading the relatively localized stress and buckling concentrations seen in the conventional case. Finally, considering the slices labelled A-D, we see further confirmation of the results discussed before, along with the mechanism by which the morphing achieves these results. Again, results for the nominal case and the 2.5-g case are shown. Considering the pressure distributions on the slices, we see typical results for most cases, except the maneuver condition with morphing. For this case, the pressure distributions on the outboard section of the wing have inverted over the morphed region. To see the cause of this, we consider the geometric slices. Shown in the upper right corner of each plot is a zoomed in view of the aft $20 \%$ of the slice. Here, we distinctly see the result of the morphing. At the maneuver condition, the morphing adds reverse camber on the outboard sections of the wing, producing the pressure distribution inversion and the inboard shift of the load distribution. This is the mechanism through which the wing 


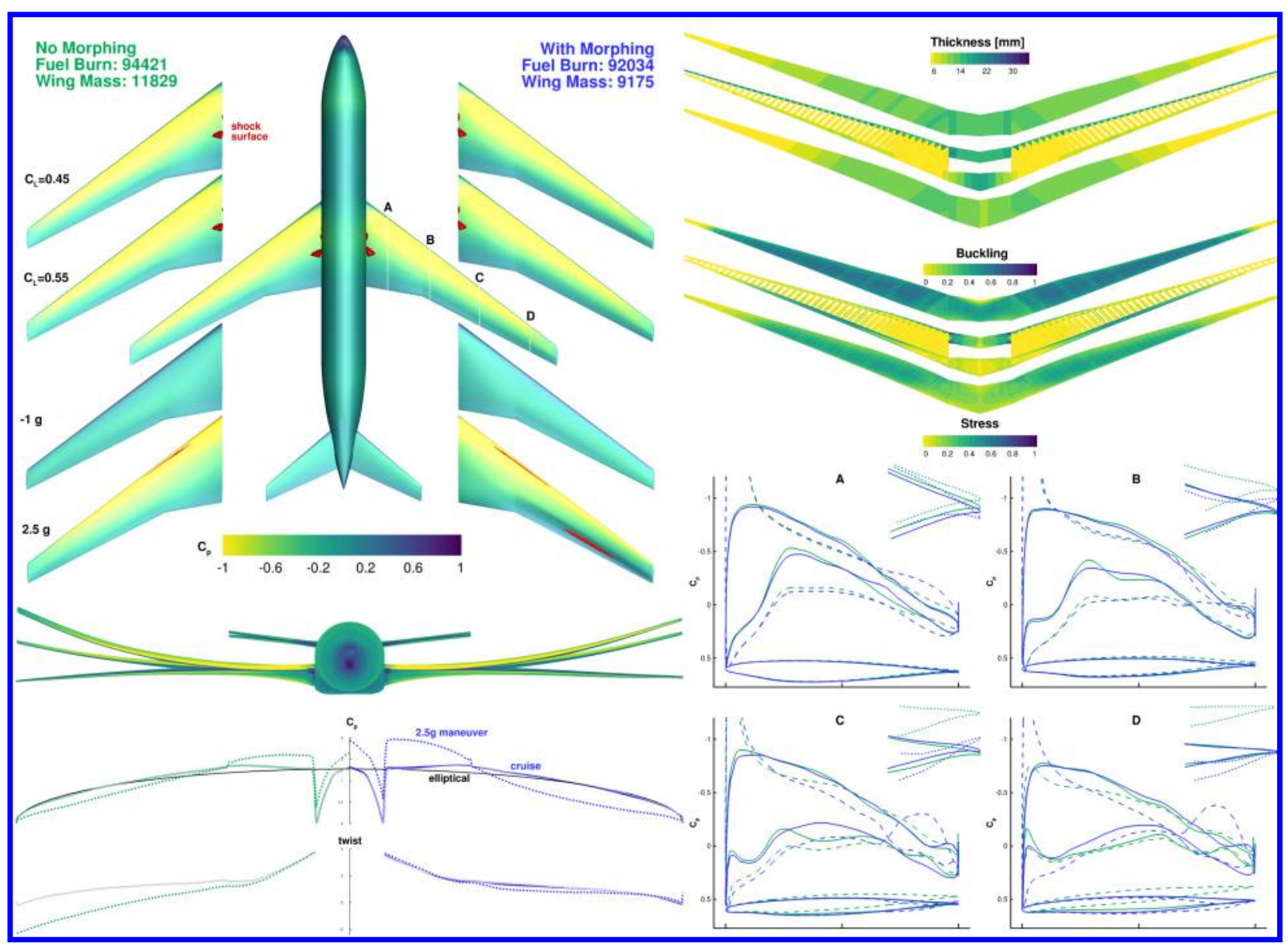

Figure 2: Adding morphing for the 3 point stencil reduced the fuel burn by $2.53 \%$, and the structural weight was reduced by $22.4 \%$. 
with morphing reduces its structural weight, rather than relying on aeroelastic coupling like the conventional wing.

We now describe the results of the same optimization applied to a 7-point stencil, shown in Figure 3. Looking at these results, we see many of the same trends as for the 3-point optimization. Again, the addition of morphing led to a substantial fuel burn reduction, this time of over 5\%, largely through the reduction of structural weight. As in the 3-point case, this reduction was enabled by the inboard shift of the maneuver load distribution resulting from the negative camber added to the outboard sections of the wing by the morphing. This mechanism for improving the aircraft's performance seems to be the same in the two morphing cases. However, by examining the results in a bit more detail, we can gather a few more insights.

Looking at the structural weights of the two wings optimized with morphing, we see that the optimal 7-point wing has a lighter structure. This is interesting because the maneuver conditions and structural constraints used in both cases were the same. That is, both structures were sized so that the wing would not buckle or fail in either the $2.5 \mathrm{~g}$ pull up or the $-1.0 \mathrm{~g}$ push over maneuvers. This discrepancy suggests that in the 7-point case, there is an increased incentive to reduce the structural weight. To understand why this is the case, we consider the objective function: the average fuel burn of the cruise condition as estimated by the Breuget range equation. According to that equation, there are effectively two methods for reducing fuel burn: improving the aerodynamic performance at the cruise conditions (as given by the lift-to-drag ratio), or reducing the structural weight of the aircraft. These are the two mechanisms a morphing trailing edge can use to improve the aircraft's fuel burn. We have already discussed the process by which morphing can reduce the structural weight. Morphing can also improve fuel burn through improvements to the aerodynamic performance at cruise. Without morphing, the wing shape is forced to compromise to achieve good performance at all of the flight conditions; however, the inclusion of morphing reduces the coupling between aerodynamic performance at various flight conditions. This was seen clearly in our previous single point work [11]. As such, the optimization of a wing with morphing for minimum fuel burn is a balance between improving aerodynamic performance at cruise and reducing structural weight. While morphing helps to reduce the coupling between flight conditions, the dependence is not completely removed, as the portion of the wing forward of the morphing is the same for all flight conditions, and the thicknesses of the wingbox members cannot change in flight. Within this context, the lower structural weight for the 7-point result provides an interesting insight. With the addition of cruise flight conditions, the balance between improving aerodynamic performance and reducing structural weight shifted towards reducing the structural weight. As there were no changes made to the constraints on the structure, this implies that the aerodynamic improvements available in the 7-point case are smaller than those available in the 3 point case. This conclusion make sense, given the coupling between aerodynamic performance caused by the nonmorphing section of the wing. Extrapolating this trend to consider aerodynamic performance for an aircraft for a full flight's worth of conditions, the reductions available due to morphing become increasingly important. While morphing provides aerodynamic improvements through adaptability at a wide range of cruise conditions, its ability to substantially reduce structural weight through adaptive maneuver load alleviation yields a lower weight structure, which reduces fuel burn at all flight conditions.

\section{B. $30 \%$ morphing device}

In this subsection, we discuss two additional optimizations, which resulted from reducing the size of the morphing device from the aft $40 \%$ of the chord to the aft $30 \%$. The problem definition and setup for the $30 \%$ optimizations was similar to the previously discussed morphing optimization, except that the number of control points with morphing freedom was reduced by half, thus limiting the size of the morphing device. These optimizations were done to gain some insight into the significance the size of the morphing region has on the effectiveness of the morphing device. Figures 4 and 5 show comparisons between the uCRM wing optimized with a 30\% and $40 \%$ morphing trailing edge for the 3-point and 7-point stencils, respectively. The results for the $40 \%$ morphing device are the same as were shown in Figures 2 and 3. Looking at the results, the reduction of the morphing region produced small increases in both the wing mass and the fuel burn. As would be expected, with a smaller morphing device the maneuver load alleviation was slightly less effective, resulting in the heavier structure and larger fuel burn. The general trends of the result match those for the $40 \%$ morphing device, again showing a lower structural weight for the 7-point case due to the previously detailed balance between reducing structural weight and improving cruise performance. While there is a reduction in savings for a smaller morphing device, the savings as compared to the wing without morphing are still sizable, showing that even if a morphing device is unable to extend all the way to the aft edge of the wingbox, it can still be an effective fuel burn reduction mechanism. 


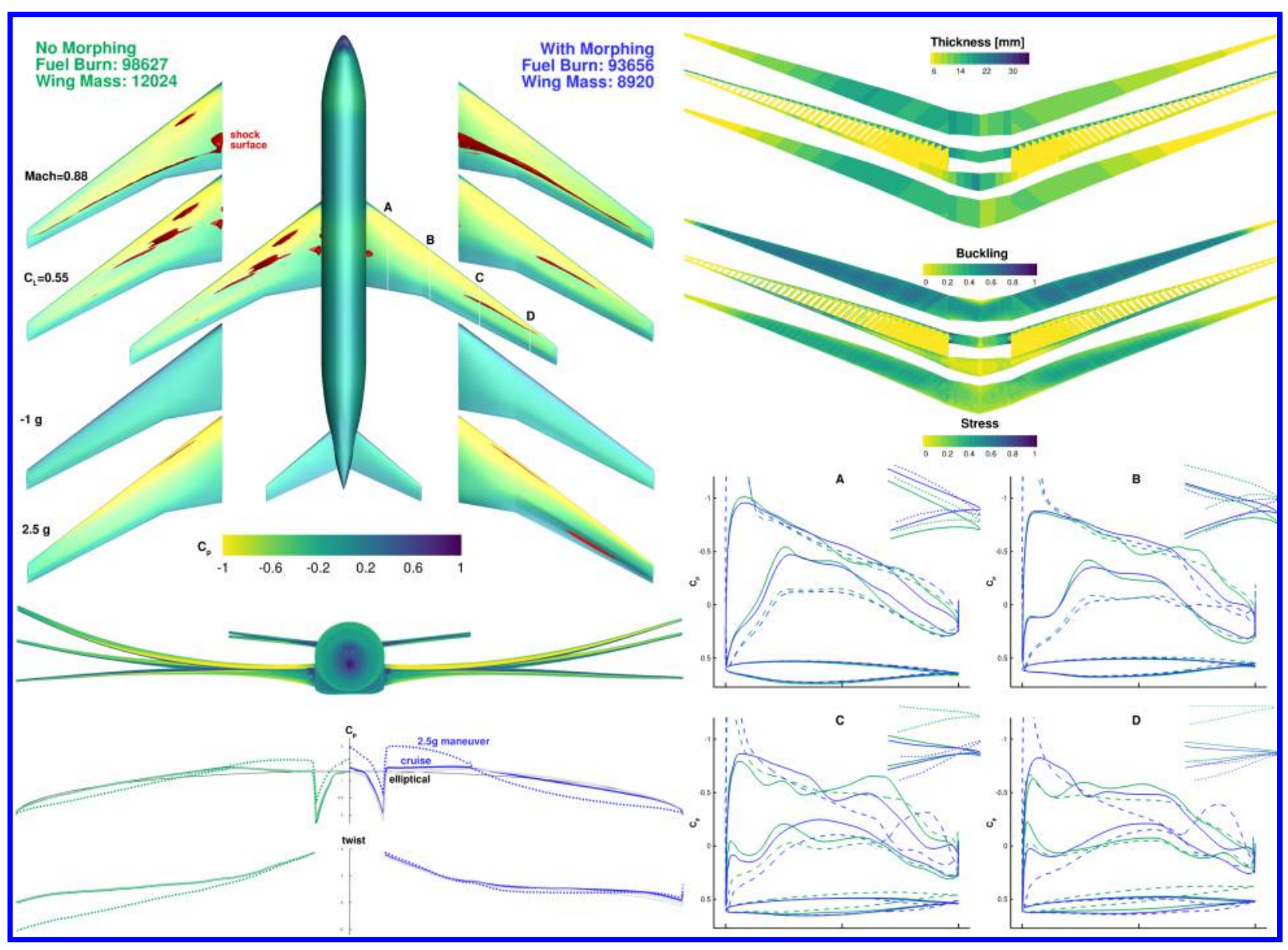

Figure 3: Adding morphing for the 7 point stencil reduced the fuel burn by $5.04 \%$, and the structural weight was reduced by $25.8 \%$. 


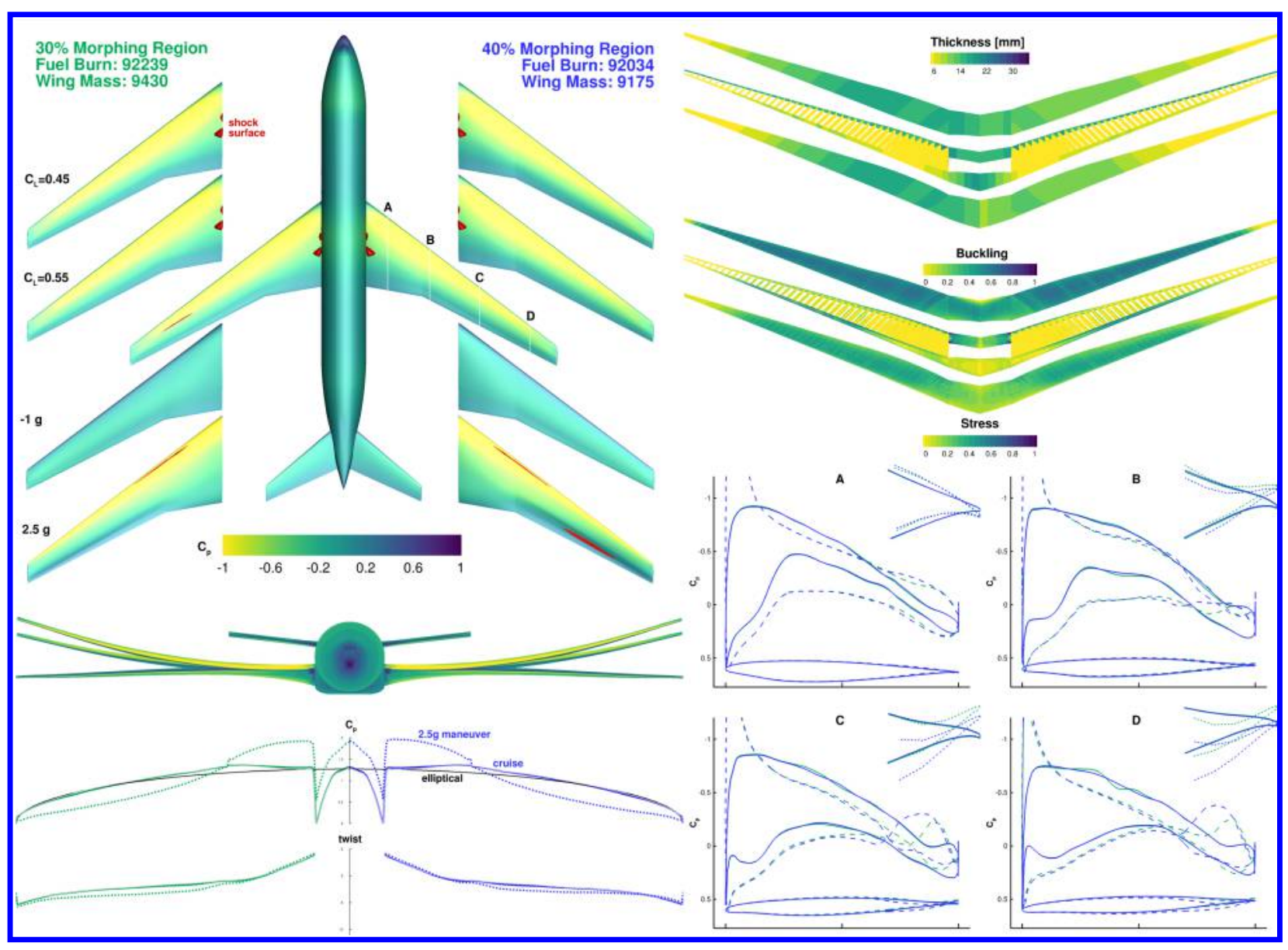

Figure 4: For the 3 point stencil when the morphing region was reduced from $40 \%$ to $30 \%$ of the chord, the fuel burn increased by $0.22 \%$ and the wing mass increased by $2.78 \%$. 


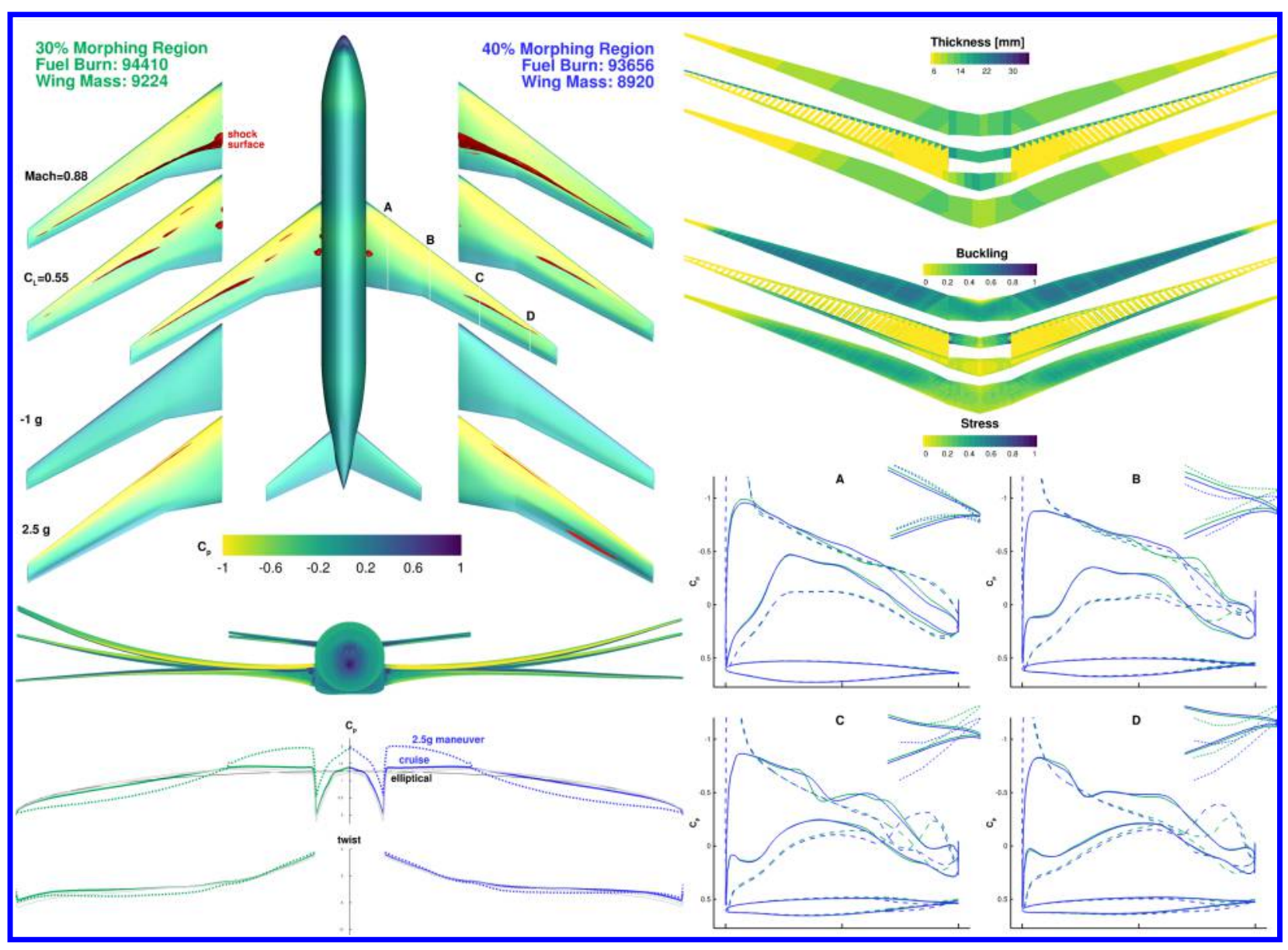

Figure 5: For the 7 point stencil when the morphing region was reduced from $40 \%$ to $30 \%$ of the chord, the fuel burn increased by $0.81 \%$ and the wing mass increased by $3.41 \%$. 


\section{High aspect ratio wing}

In this optimization, we consider a modified version of the uCRM, which has an increased aspect ratio of 13.5. Increasing the aspect ratio of a wing reduces induced drag, and is therefore aerodynamically beneficial. Conceptual designs for high aspect ratio wings are enabled by the use of modern composite materials which produce very flexible wings. Increased flexibility leads to greater differences between the shapes at various flight conditions, and therefore could negatively impact the multipoint aerodynamic performance. We demonstrated that a morphing trailing edge device can counteract this effect, improving the aerodynamic robustness of an aircraft. It follows that there is a larger potential for fuel burn savings in aircraft with larger aspect ratios. To explore this assumption, we consider a 3-point optimization much like that outlined in Section III.A. The results of that optimization are shown in Figure 6.

The optimization results confirm the assumption that morphing trailing edge devices are more effective for higher aspect ratio wings. Comparing the results in Figure 6 to those from previous optimizations, we again see many of the same trends. The morphing device produced substantial fuel burn reductions due largely to an inboard shift of the maneuver load distribution. Comparing the results in Figure 6 to those in Figure 2, we see the percentage reduction in structural weight is nearly identical ( $22.2 \%$ vs $22.4 \%$ for the high and low aspect ratio wings, respectively). However, the fuel burn reduction is more significant for the high aspect ratio case (3.79\% vs. $2.53 \%)$. It follows that in the high aspect ratio optimization the morphing device was able to provide more substantial aerodynamic improvements. As the aspect ratio increases, the robustness of conventional wings becomes limited, providing more opportunity for morphing devices to improve performance. It is clear that through maneuver load alleviation and increased aerodynamic robustness, morphing trailing edge technology can help enable higher aspect ratio wing design in future aircraft.

\section{Conclusions}

The benefits of morphing trailing edge technology were studied by performing a number of multipoint coupled aerostructural optimizations. The uCRM geometry was optimized to minimize fuel burn using conventional shape, twist, and structural thickness design variables for both a 3- and 7-point stencil. Those optimizations were then repeated with the addition of morphing shape design variables at each of the non-nominal flight conditions. Fuel burn reductions of 2.53 and $5.04 \%$ were achieved for the 3 point and seven point optimizations, respectively. These reductions were achieved through improved aerodynamic performance at the cruise conditions and reduced structural weight resulting from an inboard shift of maneuver load distributions. Further analysis revealed that as the number of points in the multipoint stencil is increased, the balance between improving aerodynamic performance and reducing structural weight shifts towards a reduction in weight. Reductions in structural weight provide fuel savings at all of the flight conditions, giving rise to the inverse relationship we observed between the number of flight conditions and the optimal structural weight. It follows that for an aircraft flying an array of flight conditions seen during a full flight or full lifetime, the ability of a morphing trailing edge to reduce structural weight is crucial to improving fuel efficiency. The efficiency of conventional tube and wing aircraft has started to plateau in recent years, but the introduction of innovative technology like morphing trailing edge devices has the potential to provide performance improvements in the near future. This technology can be particularly effective if used to enable larger aspect ratio wings, as demonstrated herein through maneuver load alleviation and increased aerodynamic robustness.

\section{Acknowledgements}

The authors extend their gratitude to their collaborators at NASA, who supported this work through award No. NNX11AI19A. This work used the Extreme Science and Engineering Discovery Environment (XSEDE), which is supported by National Science Foundation grant number ACI-1053575 [26]. 


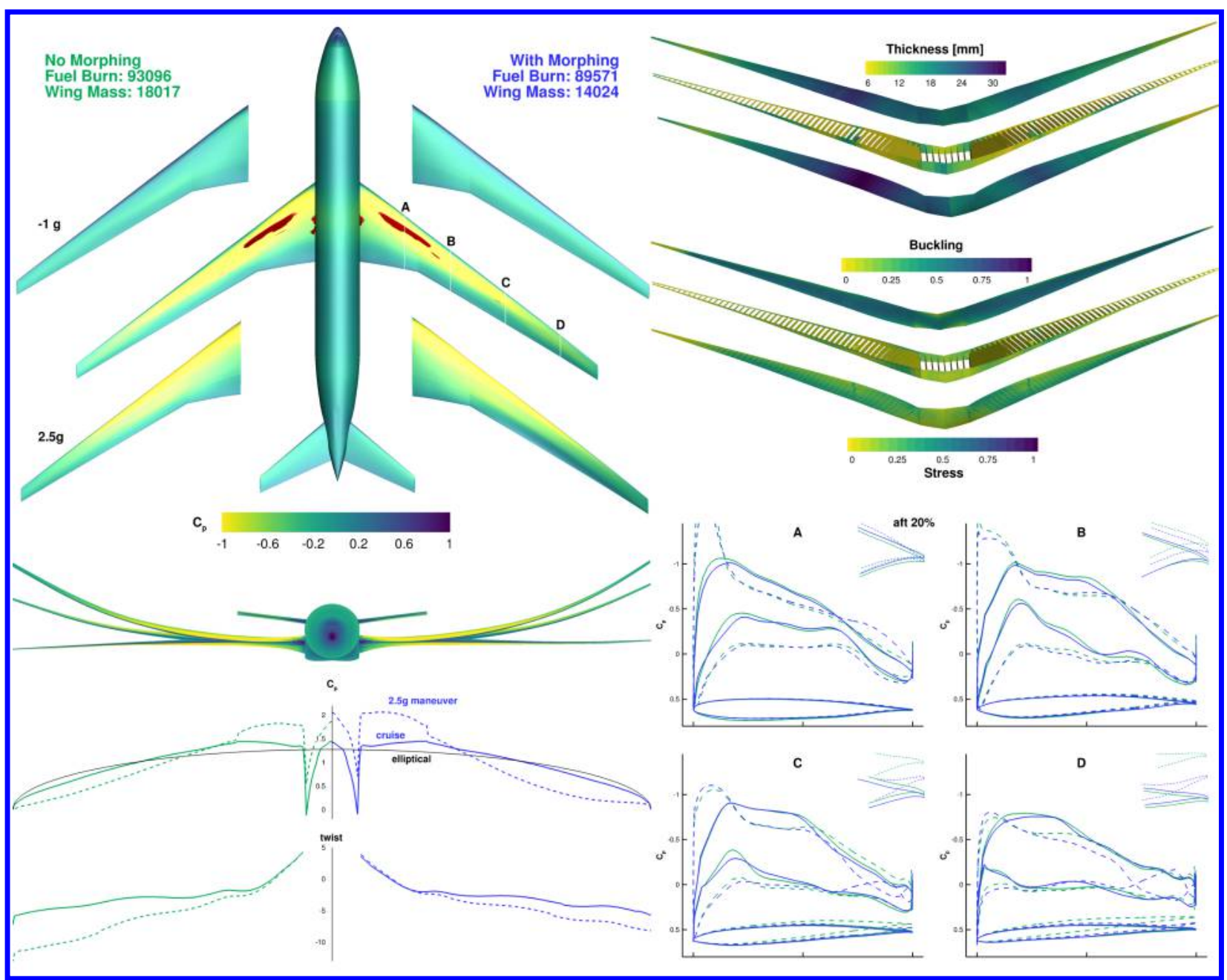

Figure 6: Adding a morphing trailing edge device to the high aspect ratio uCRM enabled a $22.2 \%$ reduction in structural weight, and produced a fuel burn savings of $3.79 \%$. 


\section{References}

[1] Jutte, C. V., Stanford, B. K., Wieseman, C. D., and Moore, J. B., "Aeroelastic tailoring of the NASA common research model via novel material and structural configurations," AIAA SciTech Conference, 2014, pp. 13-17.

-[2] Kota, S., Hetrick, J., Osborn, R., Paul, D., Pendleton, E., Flick, P., and Tilmann, C., "Design and Application of Compliant Mechanisms for Morphing Aircraft Structures," Proceedings of SPIE, Vol. 5054, 2003, p. 25.

[3] Kota, S., Osborn, R., Ervin, G., Maric, D., Flick, P., and Paul, D., "Mission Adaptive Compliant Wing-Design, Fabrication and Flight Test," RTO Applied Vehicle Technology Panel (AVT) Symposium, 2009.

-[4] Kota, S., Flick, P., and Collier, F., "Flight Testing of the FlexFloil TM Adaptive Compliant Trailing Edge," 54th AIAA Aerospace Sciences Meeting, 2016, p. 0036.

[5] Hanselka, H., “A Lightweight Concept for Aerodynamic Surfaces With Variable Camber,” 1998.

[6] Monner, H., Sachau, D., and Breitbach, E., Design aspects of the elastic trailing edge for an adaptive wing, Defense Technical Information Center, 2000.

[7] Molinari, G., Quack, M., Dmitriev, V., Morari, M., Jenny, P., and Ermanni, P., "Aero-Structural Optimization of Morphing Airfoils for Adaptive Wings,” Journal of Intelligent Material Systems and Structures, Vol. 22, No. 10, 2011, pp. 1075-1089. doi:10.1177/1045389X11414089.

- [8] Lyu, Z., Kenway, G. K., and Martins, J. R. R. A., "Aerodynamic Shape Optimization Studies on the Common Research Model Wing Benchmark,” AIAA Journal, Vol. 53, No. 4, April 2015, pp. 968-985. doi:10.2514/1.J053318.

[9] Lyu, Z. and Martins, J. R. R. A., “Aerodynamic Shape Optimization of an Adaptive Morphing Trailing Edge Wing,” Journal of Aircraft, 2015, (Accepted).

-[10] Wakayama, S. and White, E. V., "Evaluation of Adaptive Compliant Trailing Edge Technology," 33rd AIAA Applied Aerodynamics Conference, AIAA, Vol. 3289, 2015.

-[11] Burdette, D., Kenway, G. K. W., Lyu, Z., and Martins, J. R. R. A., “Aerostructural Design Optimization of an Adaptive Morphing Trailing Edge Wing," Proceedings of the AIAA Science and Technology Forum and Exposition (SciTech), Kissimmee, FL, January 2015, AIAA 2015-1129.

[12] Kenway, G. K. W., Kennedy, G. J., and Martins, J. R. R. A., "Scalable Parallel Approach for High-Fidelity Steady-State Aeroelastic Analysis and Derivative Computations," AIAA Journal, Vol. 52, No. 5, May 2014, pp. 935-951. doi:10.2514/1.J052255.

[13] Kenway, G. K., Kennedy, G. J., and Martins, J. R. R. A., "A CAD-Free Approach to High-Fidelity Aerostructural Optimization," Proceedings of the 13th AIAA/ISSMO Multidisciplinary Analysis Optimization Conference, Fort Worth, TX, Sept. 2010, AIAA 2010-9231.

-[14] Sederberg, T. W. and Parry, S. R., "Free-form Deformation of Solid Geometric Models," SIGGRAPH Comput. Graph., Vol. 20, No. 4, Aug. 1986, pp. 151-160. doi:10.1145/15886.15903.

[15] Uyttersprot, L., Inverse Distance Weighting Mesh Deformation, Ph.D. thesis, Delft University of Technology, 2014.

[16] van der Weide, E., Kalitzin, G., Schluter, J., and Alonso, J. J., "Unsteady Turbomachinery Computations Using Massively Parallel Platforms," Proceedings of the 44th AIAA Aerospace Sciences Meeting and Exhibit, Reno, NV, 2006, AIAA 20060421.

[17] Lyu, Z., Kenway, G. K., Paige, C., and Martins, J. R. R. A., “Automatic Differentiation Adjoint of the Reynolds-Averaged Navier-Stokes Equations with a Turbulence Model," 21 st AIAA Computational Fluid Dynamics Conference, San Diego, CA, Jul. 2013. doi:10.2514/6.2013-2581.

- [18] Kennedy, G. J. and Martins, J. R. R. A., "A Parallel Finite-Element Framework for Large-Scale Gradient-Based Design Optimization of High-Performance Structures," Finite Elements in Analysis and Design, Vol. 87, September 2014, pp. 56-73. doi:10.1016/j.finel.2014.04.011.

[19] Brown, S. A., "Displacement Extrapolation for CFD+CSM Aeroelastic Analysis," Proceedings of the 35th AIAA Aerospace Sciences Meeting, Reno, NV, 1997, AIAA 1997-1090.

-[20] Gill, P. E., Murray, W., and Saunders, M. A., "SNOPT: An SQP algorithm for large-scale constrained optimization," SIAM Journal of Optimization, Vol. 12, No. 4, 2002, pp. 979-1006. doi:10.1137/S1052623499350013.

[21] Perez, R. E., Jansen, P. W., and Martins, J. R. R. A., “pyOpt: A Python-Based Object-Oriented Framework for Nonlinear Constrained Optimization,” Structural and Multidisciplinary Optimization, Vol. 45, No. 1, January 2012, pp. 101-118. doi:10.1007/s00158-011-0666-3.

[22] Kenway, G. K. W., Kennedy, G. J., and Martins, J. R. R. A., “Aerostructural Optimization of the Common Research Model Configuration," 15th AIAA/ISSMO Multidisciplinary Analysis and Optimization Conference, Atlanta, GA, June 2014, AIAA 2014-3274.

-[23] Vassberg, J. C., DeHaan, M. A., Rivers, S. M., and Wahls, R. A., "Development of a Common Research Model for Applied CFD Validation Studies," 2008, AIAA 2008-6919. 
-[24] Kennedy, G. J. and Martins, J. R. R. A., "A Comparison of Metallic and Composite Aircraft Wings Using Aerostructural Design Optimization," 14th AIAA/ISSMO Multidisciplinary Analysis and Optimization Conference, Indianapolis, IN, Sep. 2012, AIAA-2012-5475.

[25] Lyu, Z. and Martins, J. R. R. A., "Aerodynamic Shape Optimization of an Adaptive Morphing Trailing Edge Wing," Proceedings of the 15th AIAA/ISSMO Multidisciplinary Analysis and Optimization Conference, Atlanta, GA, June 2014. doi:10.2514/6.2014-3275, AIAA 2014-3275.

[26] Towns, J., Cockerill, T., Dahan, M., Foster, I., Gaither, K., Grimshaw, A., Hazlewood, V., Lathrop, S., Lifka, D., Peterson, G. D., Roskies, R., Scott, J. R., and Wilkens-Diehr, N., "XSEDE: Accelerating Scientific Discovery," Computing in Science and Engineering, Vol. 16, No. 5, 2014, pp. 62-74. doi:10.1109/MCSE.2014.80. 


\section{This article has been cited by:}

1. John T. Hwang. Reconfigurable model execution in the OpenMDAO framework . [Citation] [PDF] [PDF Plus]

2. Timothy R. Brooks, Gaetan K. Kenway, Joaquim Martins. Undeflected Common Research Model (uCRM): An Aerostructural Model for the Study of High Aspect Ratio Transport Aircraft Wings . [Citation] [PDF] [PDF Plus]

3. Gaetan K. W. Kenway, Joaquim R. R. A. Martins. 2017. Buffet-Onset Constraint Formulation for Aerodynamic Shape Optimization. AIAA Journal 55:6, 1930-1947. [Abstract] [Full Text] [PDF] [PDF Plus]

4. Nitin Garg, Gaetan K.W. Kenway, Joaquim R.R.A. Martins, Yin Lu Young. 2017. High-fidelity multipoint hydrostructural optimization of a 3-D hydrofoil. Journal of Fluids and Structures 71, 15-39. [Crossref]

5. Gaetan K. Kenway, Asitav Mishra, Ney R. Secco, Karthikeyan Duraisamy, Joaquim Martins. An Efficient Parallel Overset Method for Aerodynamic Shape Optimization . [Citation] [PDF] [PDF Plus]

6. Timothy R. Brooks, Graeme Kennedy, Joaquim Martins. High-fidelity Multipoint Aerostructural Optimization of a High Aspect Ratio Tow-steered Composite Wing . [Citation] [PDF] [PDF Plus]

7. Bret K. Stanford. 2016. Static and Dynamic Aeroelastic Tailoring with Variable-Camber Control. Journal of Guidance, Control, and Dynamics 39:11, 2522-2534. [Abstract] [Full Text] [PDF] [PDF Plus]

8. David A. Burdette, Gaetan K. Kenway, Joaquim Martins. Aerostructural design optimization of a continuous morphing trailing edge aircraft for improved mission performance . [Citation] [PDF] [PDF Plus]

9. John Hwang, Joaquim Martins. Allocation-mission-design optimization of next-generation aircraft using a parallel computational framework . [Citation] [PDF] [PDF Plus] 